21

\title{
Нелинейная динамика параметрических осцилляций поляритонов в микрорезонаторе
}

\author{
() О.Ф. Васильева ${ }^{1}$, А.П. Зинган ${ }^{1}$, П.И. Хаджи ${ }^{1,2}$ \\ ${ }^{1}$ Приднестровский государственный университет им. Т.Г. Шевченко, \\ МД 3300 Тирасполь, Молдова \\ ${ }^{2}$ Институт прикладной физики АН Молдовы, \\ МД 2800 Кишинев, Молдова \\ e-mail: florina_of@mail.ru
}

Поступила в редакцию 26.03.2018 г.

В окончательной редакции 16.04.2018 г.

\begin{abstract}
Изучена динамика поляритонов в микрорезонаторе в режиме параметрического осциллятора, когда два поляритона накачки превращаются в поляритоны сигнальной и холостой мод и обратно. При этом накачка осуществляется двумя лазерами с близкими частотами. Получены аналитические решения системы нелинейных дифференциальных уравнений. Найдены периодические и апериодические режимы превращения пары поляритонов накачки в поляритоны сигнальной и холостой мод.
\end{abstract}

DOI: $10.21883 /$ OS.2018.09.46562.87-18

\section{Введение}

Смешанные экситон-фотонные состояния в плоских полупроводниковых микрорезонаторах с квантовыми ямами в активном слое представляют собой новый класс квазидвумерных квазичастиц с уникальными свойствами [1-13]. Такие состояния называют микрорезонаторными экситон-поляритонами. Они возникают благодаря сильной связи экситонов с собственными модами электромагнитного излучения микрорезонатора. В условиях сильной связи экситонная и фотонная моды расталкиваются и возникают верхняя и нижняя микрорезонаторные поляритонные моды. Экситонная компонента поляритона отвечает за эффективное поляритон-поляритонное взаимодействие, благодаря чему они могут рассеиваться друг на друге, а фотонная компонента обусловливает его малую эффективную массу. Непараболичность нижней поляритонной ветви допускает возникновение параметрического процесса, в результате которого два поляритона накачки рассеиваются в сигнальную и холостую моды с сохранением энергии и импульса. Поэтому огромный интерес вызывает поляритон-поляритонное рассеяние, благодаря которому экситон-поляритонная система демонстрирует сильно нелинейные свойства [6-13]. Такие нелинейности были обнаружены в спектрах люминесценции микрорезонаторов [14-18] при резонансном возбуждении нижней поляритонной ветви, которые объяснялись четырехволновым смешением или параметрическим рассеянием фотовозбужденных поляритонов накачки в сигнальную и холостую моды. Экспериментально идентифицированы два механизма нелинейности - поляритонное параметрическое рассеяние $[6,19,20]$ и голубой сдвиг поляритонной дисперсии $[2,5]$. С использованием pump-probe-метода в $[8,9]$ впервые наблюдалось параметрическое усиление в микрорезонаторе при возбуждении нижней поляритонной ветви пикосекундным импульсом накачки под углом падения $16.5^{\circ}$. После возбуждения (с небольшой задержкой) нижней поляритонной ветви дополнительно слабым пробным импульсом, падавшим нормально, обнаружилось, что этот импульс в отражении усиливался более чем в 70 раз. При этом появлялась также холостая мода под углом в $35^{\circ}$. Именно для этих углов выполнялись резонансные условия. Результаты экспериментов [8,9] были воспроизведены также в [21] и моделировались в [7] с использованием механизма поляритон-поляритонного рассеяния. Аналогичные процессы наблюдались в [22] при использовании двух пучков накачки под углами в $\pm 45^{\circ}$ и пробного пучка под углом $0^{\circ}$. Режим параметрического осциллятора наблюдался в $[9,14]$ при непрерывном возбуждении нижней поляритонной ветви излучением накачки под „магическим“ углом в $16^{\circ}$ без пробного импульса. Выше пороговой интенсивности наблюдались сильные пучки сигнальной и холостой мод под углами $0^{\circ}$ и $35^{\circ}$ соответственно. В [20] обнаружена сильная и необычная зависимость поляризации света, излучаемого микрорезонатором, от поляризации накачки. Эта зависимость интерпретируется с использованием псевдоспиновой модели в рамках квазиклассического формализма, где параметрическое рассеяние описывается как резонансное четырехволновое смешение. В [23-25] наблюдалось бистабильное пропускание излучения в зависимости от интенсивности накачки при возбуждении экситон-поляритонов в микрорезонаторе. Отметим, что процесс параметрического рассеяния наблюдался как при импульсном $[20,26]$, так и при непрерывном [14,16,27] возбуждениях.

Описание поляритонных параметрических осцилляторов и усилителей представлено в ряде работ $[2,5,7,8$, 12-14,17,18,28-31]. В [5] выведены квантовые кинетические уравнения, описывающие систему взаимодействую- 
щих поляритонов, которые затем применялись для изучения динамики поляритонных параметрических осцилляторов. В [7] в рамках трехуровневой модели изучались динамика волны накачки, а также сигнальной и холостой мод, которые считались когерентными и макрозаполнеными. В [5] это рассмотрение было расширено на случай учета флуктуаций полей. Полуклассическое рассмотрение базировалось на тех же методах, которые были использованы при исследовании явления четырехволнового смешения. Поляритон-поляритонные взаимодействия при этом приводили к нелинейностям третьего порядка в уравнениях Максвелла-Блоха. Этот метод приводит к системе уравнений, подобной полученной в [7]. Для исследования свойств поляритонного осциллятора в $[1,4,5]$ развит гиперспиновый формализм. Введение гиперспина позволило авторам получить квазиклассическое решение уравнений и показать, что вероятность распределения компонент гиперспина подчиняется уравнению Лиувилля. Показано также, что плотности поляритонов в сигнальной и холостой модах, осциллируя, затухают во времени. Отметим также, что в [32] теоретически анализируются явления типа джозефсон-эффекта для экситон-поляритонных конденсатов с учетом спиновых степеней свободы. Когерентные осцилляции населенности и фазы экситон-поляритонов в микрорезонаторе (бозонный эффект Джозефсона) наблюдались экспериментально в [33].

\section{Постановка задачи. Основные уравнения}

Цель настоящей работы - изучить изменение во времени плотности поляритонов при накачке нижней ветви в двух близких по энергии точках закона дисперсии. В [34-38] при исследовании свойств оптического параметрического осциллятора использовались два одинаковых фотона накачки на нижней ветви поляритонного закона дисперсии. Однако в [39,40] было показано, что два различных пучка накачки можно конвертировать в два вырожденных пучка на частоте фотонов сигнальной и холостой мод. Наличие двух различных пучков накачки дает большие возможности для генерации сигнального и холостого пучков с наперед заданными свойствами. Поэтому далее будем считать, что оба пучка накачки различаются по амплитуде (интенсивности), однако энергии фотонов различаются слабо. В этом случае возможны дополнительные режимы, например режим заданной плотности фотонов одного из пучков. Рассматриваем ситуацию на временах порядка либо меньших времени релаксации возбуждений среды. Мы считаем, что с помощью ультракоротких импульсов резонансного лазерного излучения в микрорезонаторе создается система когерентных экситон-поляритонов. Микрорезонатор обеспечивает пространственное ограничение области существования поляритонов, которые взаимно превращаются друг в друга. Квантовая яма,

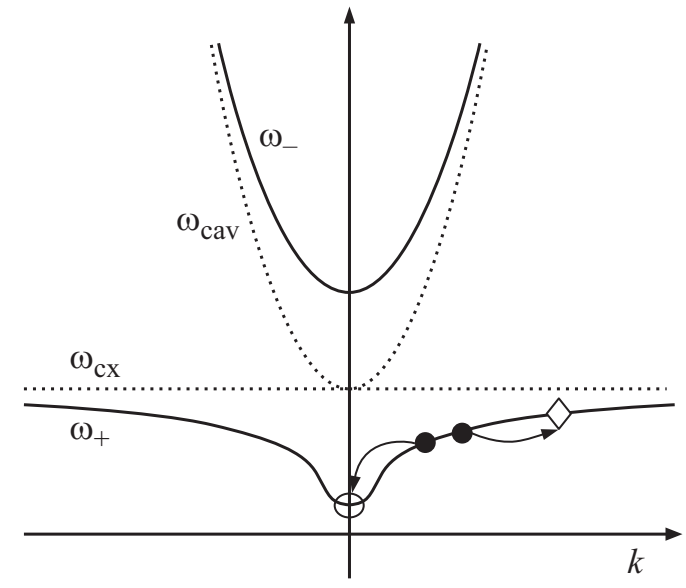

Рис. 1. Энергии поляритонов верхней и нижней ветвей $\left(\omega_{ \pm}\right)$. Дисперсия собственных частот микрорезонатора $\omega_{\text {cav }}$ и экситона $\omega_{\text {ex. }}$ Два поляритона накачки рассеиваются в сигнальную и холостую моды.

в которой возникают поляритоны, вставляется в брэгговскую структуру, которая характеризуется определенным пропусканием, отражением и потерями $[8,9,12,15,27]$. Особенности эволюции системы будут проявляться в генерации либо вторичных субимпульсов, либо периодического излучения на частотах экситон-поляритонов.

Ниже рассматриваем ситуацию, когда поляритоны возбуждаются на нижней ветви закона дисперсии под „магическим“ углом (рис. 1). В [4,5] показано, что процесс параметрического рассеяния двух поляритонов накачки в сигнальную и холостую моды описывается гамильтонианом вида

$$
\begin{aligned}
\frac{1}{\hbar} H= & \omega_{p_{1}} \hat{a}_{p_{1}}^{+} \hat{a}_{p_{1}}+\omega_{p_{2}} \hat{a}_{p_{2}}^{+} \hat{a}_{p_{2}}+\omega_{s} \hat{a}_{s}^{+} \hat{a}_{s}+\omega_{i} \hat{a}_{i}^{+} \hat{a}_{i} \\
& +\mu\left(\hat{a}_{s}^{+} \hat{a}_{i}^{+} \hat{a}_{p_{1}} \hat{a}_{p_{2}}+\hat{a}_{p_{1}}^{+} \hat{a}_{p_{2}}^{+} \hat{a}_{s} \hat{a}_{i}\right)
\end{aligned}
$$

где $\omega_{p_{1}}, \omega_{p_{2}}, \omega_{s}$ и $\omega_{i}-$ собственные частоты поляритонов накачки, сигнальной и холостой мод соответственно, $\hat{a}_{p_{1}}, \hat{a}_{p_{2}}, \hat{a}_{s}, \hat{a}_{i}$ - операторы уничтожения поляритонов, $\mu$ - константа параметрической поляритон-поляритонной конверсии. Используя (1), легко получить систему гайзенберговских уравнений для операторов $\hat{a}_{p_{1}}, \hat{a}_{p_{2}}$, $\hat{a}_{s}, \hat{a}_{i}$. Усредняя эту систему уравнений и используя приближение среднего поля (mean field approximation), применимость которого обоснована в [41], в условиях точного резонанса $\left(\omega_{p_{1}}+\omega_{p_{2}}-\omega_{s}-\omega_{i}=0\right)$ для комплексных амплитуд поляритонов $a_{p_{1}}=\left\langle\hat{a}_{p_{1}}\right\rangle, a_{p_{2}}=\left\langle\hat{a}_{p_{2}}\right\rangle$, $a_{s}=\left\langle\hat{a}_{s}\right\rangle$ и $a_{i}=\left\langle\hat{a}_{i}\right\rangle$ можно получить следующую систему нелинейных эволюционных уравнений:

$$
\begin{aligned}
& i \dot{a}_{p_{1}}=\mu a_{p_{2}}^{*} a_{s} a_{i}, \quad i \dot{a}_{p_{2}}=\mu a_{p_{1}}^{*} a_{s} a_{i}, \\
& i \dot{a}_{s}=\mu a_{p_{1}} a_{p_{2}} a_{i}^{*}, \quad i \dot{a}_{i}=\mu a_{p_{1}} a_{p_{2}} a_{s}^{*} .
\end{aligned}
$$



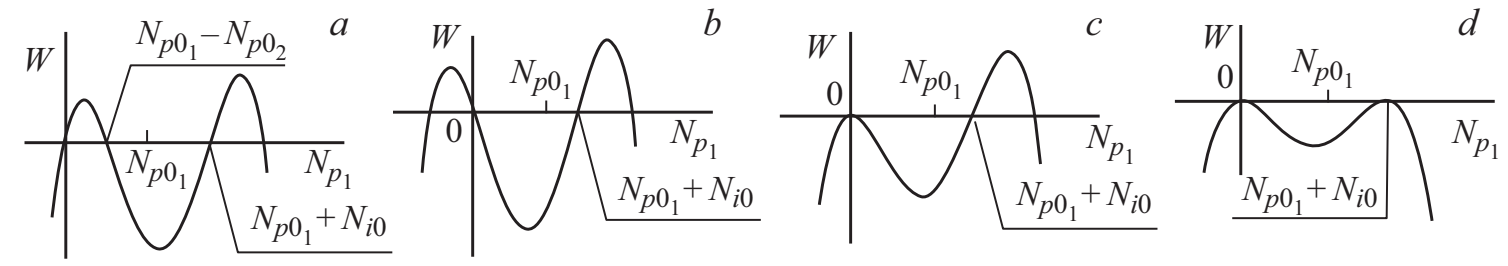

Рис. 2. Зависимость потенциальной энергии $W$ от нормированной плотности поляритонов накачки $N_{p_{1}}$ при различных соотношениях между начальными плотностями поляритонов: (a) $N_{p 0_{1}}>N_{p 0_{2}}$, (b) $N_{p 0_{1}}<N_{p 0_{2}},(c) N_{p 0_{1}}=N_{p 0_{2}}$, (d) $N_{p 0_{1}}=N_{p 0_{2}}$ и $N_{s 0}=N_{i 0}$.

Решения уравнений (2) ищем в виде

$$
\begin{gathered}
a_{p_{1}}=A_{p_{1}} \exp \left(i \varphi_{p_{1}}\right), \quad a_{p_{2}}=A_{p_{2}} \exp \left(i \varphi_{p_{2}}\right), \\
a_{s}=A_{s} \exp \left(i \varphi_{s}\right), \quad a_{i}=A_{i} \exp \left(i \varphi_{i}\right),
\end{gathered}
$$

где $A_{p_{1}}, A_{p_{2}}, A_{s}, A_{i}$ и $\varphi_{p_{1}}, \varphi_{p_{2}}, \varphi_{s}, \varphi_{i}$ - действительные амплитуды и фазы соответствующих поляритонных состояний. В результате для амплитуд и разности фаз $\theta=\varphi_{p_{1}}+\varphi_{p_{2}}-\varphi_{s}-\varphi_{i}$ получаем систему нелинейных эволюционных уравнений:

$$
\begin{gathered}
\dot{A}_{p_{1}}=-\mu A_{p_{2}} A_{s} A_{i} \sin \theta, \quad \dot{A}_{p_{2}}=-\mu A_{p_{1}} A_{s} A_{i} \sin \theta, \\
\dot{A}_{s}=\mu A_{p_{1}} A_{p_{2}} A_{i} \sin \theta, \quad \dot{A}_{i}=\mu A_{p_{1}} A_{p_{2}} A_{s} \sin \theta, \\
\dot{\theta}=\mu\left(\frac{A_{p_{1}} A_{p_{2}} A_{i}}{A_{s}}+\frac{A_{p_{1}} A_{p_{2}} A_{s}}{A_{i}}-\frac{A_{p_{1}} A_{s} A_{i}}{A_{p_{2}}}-\frac{A_{p_{2}} A_{s} A_{i}}{A_{p_{1}}}\right) \cos \theta .
\end{gathered}
$$

Систему уравнений (3) дополним начальными условиями $A_{p_{1} \mid t=0}=A_{p 0_{1}}, A_{p_{2} \mid t=0}=A_{p 0_{2}}, A_{s \mid t=0}=A_{s 0}, A_{i \mid t=0}=A_{i 0}$ и $\theta_{\mid t=0}=\theta_{0}$.

Из (3) легко получить следующие интегралы движения:

$$
\begin{gathered}
A_{p_{1}}^{2}-A_{p_{2}}^{2}=A_{p 0_{1}}^{2}-A_{p 0_{2}}^{2}, \quad A_{s}^{2}-A_{i}^{2}=A_{s 0}^{2}-A_{i 0}^{2} . \\
A_{p_{1}}^{2}+A_{s}^{2}=A_{p 0_{1}}^{2}+A_{s 0}^{2}, \quad A_{p_{2}}^{2}+A_{i}^{2}=A_{p 0_{2}}^{2}+A_{i 0}^{2}, \\
\cos \theta=\frac{A_{p 0_{1}} A_{p 0_{2}} A_{s 0} A_{i 0}}{A_{p_{1}} A_{p_{2}} A_{s} A_{i}} \cos \theta_{0} .
\end{gathered}
$$

Вводя далее плотности поляритонов $N_{p_{1}}=A_{p_{1}}^{2}$, $N_{p_{2}}=A_{p_{2}}^{2}, N_{s}=A_{s}^{2}, N_{i}=A_{i}^{2}$ и используя полученные интегралы движения, систему уравнений (4) можно привести к одному нелинейному дифференциальному уравнению, описывающему временную эволюцию плотности поляритонов накачки $N_{p_{1}}$ :

$$
\dot{N}_{p_{1}}^{2}+W\left(N_{p_{1}}\right)=E_{0}
$$

где

$$
\begin{gathered}
W\left(N_{p_{1}}\right)=-N_{p_{1}}\left(N_{p 0_{2}}-N_{p 0_{1}}+N_{p_{1}}\right) \\
\times\left(N_{s 0}+N_{p 0_{1}}-N_{p_{1}}\right)\left(N_{i 0}+N_{p 0_{1}}-N_{p_{1}}\right), \\
E_{0}=N_{p 0_{1}} N_{p 0_{2}} N_{s 0} N_{i 0} \cos ^{2} \theta_{0} .
\end{gathered}
$$

Здесь $W\left(N_{p_{1}}\right)$ играет роль потенциальной энергии, $\dot{N}_{p_{1}}^{2}-$ кинетической энергии, $E_{0}-$ полной энергии нелинейного осциллятора.

На рис. 2 представлен график зависимости потенциальной энергии нелинейного осциллятора от $N_{p_{1}}$ при различных соотношениях между начальными плотностями поляритонов. Видно, что при $N_{p 0_{1}} \neq N_{p 0_{2}}$ (рис. $2, a, b$ ) имеет место периодическая эволюция плотностей поляритонов, что и подтверждает исходное предположение о характере эволюции системы под действием двух различных пучков накачки. При $N_{p 0_{1}}=N_{p 0_{2}}$ и $E_{0}=0$ (рис. 2,c) эволюция системы является апериодической. То же самое имеет место при дополнительном условии $N_{s 0}=N_{i 0}$ (pис. 2, $d$ ).

Использование двух накачек открывает дополнительные возможности при исследовании особенностей динамики поляритонов путем существенного увеличения интенсивности одной из накачек по сравнению с другой. Видно, что при $N_{p 0_{2}}>N_{p 0_{1}}$ и $E_{0}=0$ (рис. $2, b$ ) изображающая точка может перемещаться между двумя точками поворота классической траектории $N_{p_{1}}=0$ и $N_{p_{1}}=N_{p 0_{1}}+N_{i 0}$, которые являются корнями уравнения $W\left(N_{p_{1}}\right)=0$. Следовательно, временная эволюция плотности поляритонов $N_{p_{1}}(t)$ будет представлять собой нелинейные периодические колебания. При $N_{p 0_{2}}=N_{p 0_{1}}$ (рис. 2,c) левый максимум потенциальной энергии смещается в точку $N_{p_{1}}=0$ и уравнение $W\left(N_{p_{1}}\right)=0$ имеет двукратно вырожденный корень $N_{p_{1}}=0$. В этом случае возможен только апериодический режим эволюции. Наконец, при $N_{p 0_{1}}>N_{p 0_{2}}$ (рис. $\left.2, a\right)$ уравнение $W\left(N_{p_{1}}\right)=0$ имеет четыре действительных корня. Эволюция системы является периодической. Функция $N_{p_{1}}(t)$ изменяется в пределах $N_{p 0_{1}}-N_{p 0_{2}} \leq N_{p_{1}}(t) \leq N_{p 0_{1}}+N_{i 0}$ (рис. $2, a)$. Если $E_{0} \neq 0$, то при $N_{p 0_{2}} \leq N_{p 0_{1}}$ уравнение $W\left(N_{p_{1}}\right)=E_{0}$ имеет четыре действительных корня, один из которых является отрицательным и, следовательно, функция $N_{p_{1}}(t)$ характеризуется нелинейными периодическими осцилляциями. Значения корней уравнения $W\left(N_{p_{1}}\right)=E_{0}$ определяются начальными условиями. Поэтому необходимо исследовать временную эволюцию системы при различных соотношениях между параметрами $N_{p 0_{1}}, N_{p 0_{2}}, N_{s 0}, N_{i 0}$ и $\theta_{0}$.

Рассмотрим сначала простой (и наглядный) режим эволюции системы в приближении заданной плотности поляритонов одной из компонент накачки, например, 


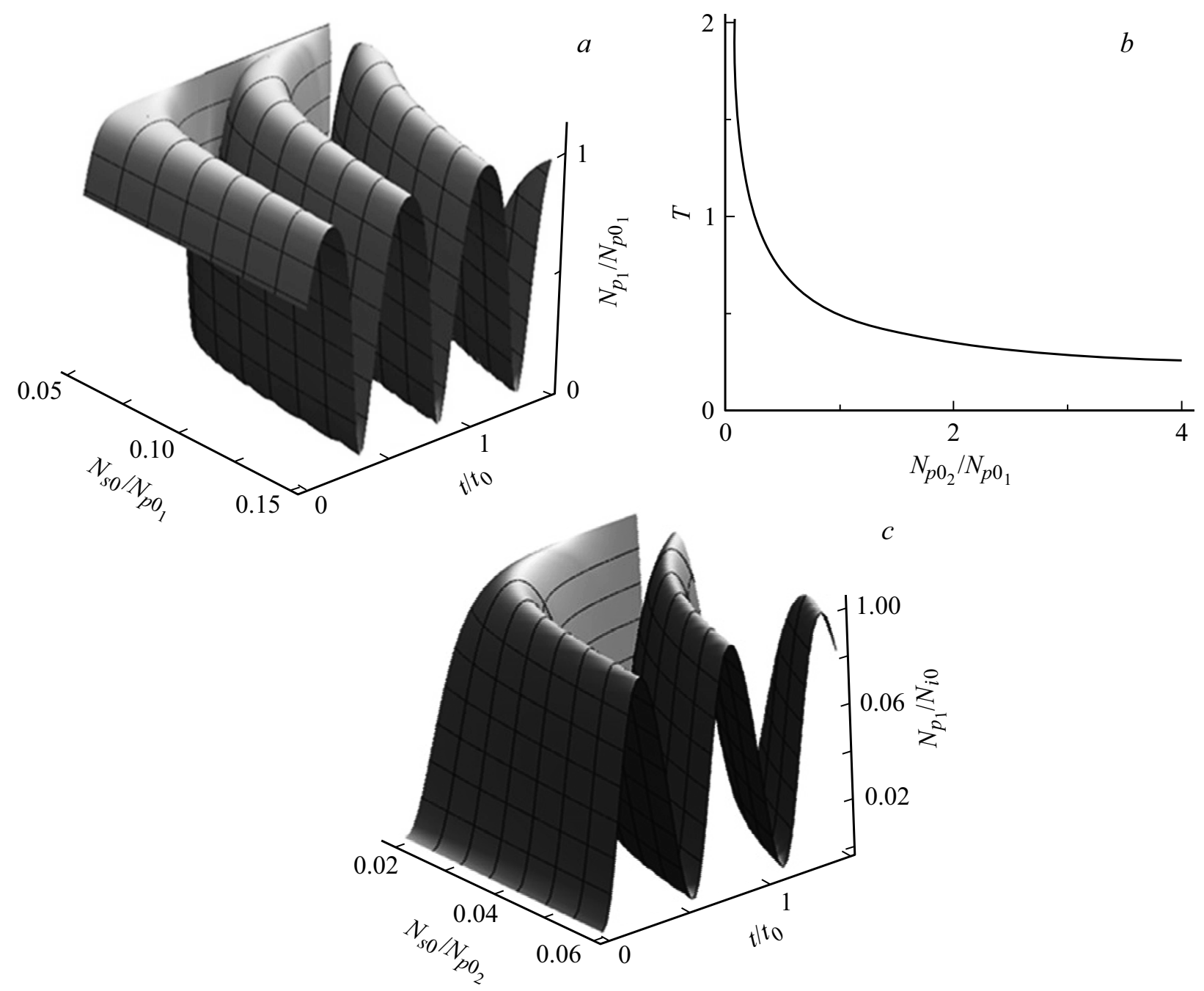

Рис. 3. Временная эволюция нормированной плотности поляритонов накачки $N_{p_{1}} / N_{p 0_{1}}$ в зависимости от отношения начальных концентраций $N_{s 0} / N_{p 0_{1}}$, где $t_{0}=\frac{\mu t}{N_{p 0_{1}}}$, при $(a) N_{p 0_{1}} \neq 0, N_{p 0_{2}} / N_{p 0_{1}}=3.5$ и $N_{i 0} / N_{p 0_{1}}=0.05 ;(c) N_{p 0_{1}}=0, N_{i 0} / N_{p 0_{2}}=0.02$. $(b)$ Период колебаний $T$ в зависимости от отношения начальных концентраций $N_{p 0_{2}} / N_{p 0_{1}}$.

$N_{p 0_{2}} \gg N_{p 0_{1}}$. В этом приближении при $N_{s 0}>N_{i 0}$ и $\theta_{0}=\frac{\pi}{2}$ получаем следующее решение уравнения:

$N_{p_{1}}=\left(N_{p 0_{1}}+N_{i 0}\right) s n^{2}\left( \pm \mu \sqrt{N_{p 0_{2}}\left(N_{p 0_{1}}+N_{s 0}\right) t}+F\left(\varphi_{0}, k\right)\right)$,

где модуль $k$ и параметр $\varphi_{0}$ эллиптической функции $\operatorname{sn}(x)$ и $F\left(\varphi_{0}, k\right)[42,43]$ определяются выражениями

$$
k^{2}=\frac{N_{p 0_{1}}+N_{i 0}}{N_{p 0_{1}}+N_{s 0}}, \quad \varphi_{0}=\arcsin \sqrt{\frac{N_{p 0_{1}}}{N_{p 0_{1}}+N_{i 0}}} .
$$

Отсюда видно, что плотность поляритонов накачки $N_{p 0_{2}}$ осциллирует во времени с амплитудой $A$ и периодом $T$, равными

$$
\begin{gathered}
A=N_{p 0_{1}}+N_{i 0} \\
T=2 K(k) /\left(\mu \sqrt{N_{p 0_{2}}\left(N_{p 0_{1}}+N_{s 0}\right)}\right) .
\end{gathered}
$$

C ростом начальной плотности поляритонов $N_{p 0_{2}}$ период монотонно убывает (пропорционально $N_{p 0_{2}}^{-1 / 2}$ ) (рис. $3, b)$. Что касается $N_{p_{1}}$, т. е. меньшей из двух плотностей накачки, то с ростом $N_{p 0_{1}}$ амплитуда колебаний функции $N_{p_{1}}(t)$ линейно растет, период колебаний монотонно убывает, а при $N_{p 0_{2}} \rightarrow N_{p 0_{1}}$ эволюция становится апериодической. За период плотность поляритонов изменяется от нуля до $N_{p 0_{1}}+N_{i 0}$. Таким образом, меньшая из накачек полностью истощается, преобразуясь в поляритоны сигнальной и холостой мод (рис. 3,a). В пределе $N_{p 0_{2}} \gg N_{p 0_{1}}$, полагая (одна из накачек в начальный момент времени отсутствует), получаем

$$
\begin{gathered}
N_{p_{1}}=N_{i 0} s n^{2}\left(\mu \sqrt{N_{p 0_{2}} N_{s 0}} t\right), \\
k^{2}=N_{i 0} / N_{s 0} .
\end{gathered}
$$

Из (11) видно, что большая из плотностей накачки определяет период колебаний, а меньшая — амплитуду. 
С ростом $N_{p_{1}}(t)$ период колебаний функции $N_{p_{1}}(t)$ убывает, а амплитуда колебаний $N_{i 0}$ сохраняется (рис. $3, c$ ).

Если начальные плотности сигнальной и холостой мод одинаковы $\left(N_{s 0}=N_{i 0}\right)$, то решение $(8)$ принимает вид

$$
\begin{aligned}
& N_{p_{1}}=\left(N_{p 0_{1}}+N_{i 0}\right) \\
& \times\left(\frac{\sqrt{N_{p 0_{1}}} \pm \sqrt{N_{p 0_{1}}+N_{i 0}} \operatorname{th}\left(\mu t \sqrt{N_{p 0_{2}}\left(N_{p 0_{1}}+N_{i 0}\right)}\right)}{\sqrt{N_{p 0_{1}}+N_{i 0}} \pm \sqrt{N_{p 0_{1}} \operatorname{th}}\left(\mu t \sqrt{N_{p 0_{2}}\left(N_{p 0_{1}}+N_{i 0}\right)}\right)}\right)^{2} .
\end{aligned}
$$

Из (12) и рис. 4 видно, что решение со знаком $(+)$ монотонно растет со временем от значения $N_{p_{1}}=$ $=N_{p 0_{2}}$, асимптотически стремясь к предельному значению $N_{p_{1}}=N_{p 0_{1}}+N_{i 0}$. Следовательно, поляритоны сигнальной и холостой мод за время эволюции полностью превращаются в поляритоны накачки, чем эволюция и завершается. Решение со знаком $(-)$ сначала монотонно убывает со временем, обращается в нуль в момент времени

$$
t=t_{1}=\operatorname{arth} \sqrt{\frac{N_{p 0_{1}}}{N_{p 0_{1}}+N_{i 0}}} /\left(\mu \sqrt{N_{p 0_{2}}\left(N_{p 0_{1}}+N_{i 0}\right)}\right),
$$

после чего начинает расти и асимптотически стремится к тому же предельному значению $N_{p 0_{1}}+N_{i 0}$. Таким образом, на больших временах $\left(t \gg 1 /\left(\sqrt{N_{p 0_{2}}\left(N_{p 0_{1}}+N_{p 0_{2}}\right)}\right)\right)$ решения со знаком $(+)$ и со знаком (-) для плотности поляритонов $N_{p_{1}}$ асимптотически стремятся к предельному значению $N_{p 0_{1}}+N_{i 0}$ (рис. 4).

Если рассматривать случай $N_{p 0_{2}}>N_{p 0_{1}}$ и $N_{s 0}>N_{i 0}$, то решение уравнения (5) запишется в виде

$$
\begin{aligned}
N_{p_{1}}= & \frac{\left(N_{p 0_{1}}-N_{p 0_{2}}\right)\left(N_{p 0_{1}}+N_{i 0}\right)}{N_{p 0_{2}}-N_{p 0_{1}}}, \\
& \times s n^{2}\left( \pm \sqrt{\left(N_{p 0_{1}}+N_{s 0}\right)\left(N_{p 0_{2}}+N_{i 0}\right)} \mu t+F\left(\varphi_{0}, k\right)\right)
\end{aligned}
$$

где величины $k$ и $\varphi$ выражаются формулами

$$
\begin{aligned}
k^{2} & =\frac{\left(N_{p 0_{2}}+N_{s 0}\right)\left(N_{p 0_{1}}+N_{i 0}\right)}{\left(N_{p 0_{1}}+N_{s 0}\right)\left(N_{p 0_{2}}+N_{i 0}\right)}, \\
\varphi_{0} & =\arcsin \sqrt{\frac{\left(N_{p 0_{2}}+N_{i 0}\right) N_{p 0_{1}}}{\left(N_{p 0_{1}}+N_{i 0}\right) N_{p 0_{2}}}} .
\end{aligned}
$$

Период $T$ колебаний плотности поляритонов накачки выражается формулой

$$
T=2 K(k) /\left(\mu \sqrt{\left(N_{p 0_{1}}+N_{s 0}\right)\left(N_{p 0_{2}}+N_{i 0}\right)}\right),
$$

где $K(k)$ - полный эллиптический интеграл $[42,43]$. Эволюция системы в этом случае является периодической и плотность поляритонов накачки изменяется в пределах $0<N_{p 0_{1}}<N_{p 0_{1}}+N_{i 0}$.

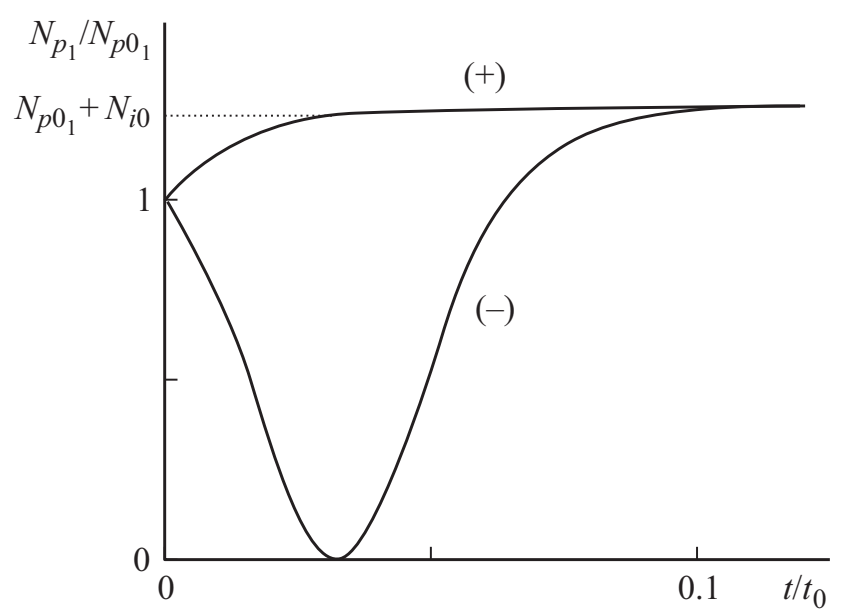

Рис. 4. Временная эволюция нормированной плотности поляритонов накачки $N_{p_{1}} / N_{p 0_{1}}$ при $N_{i 0} / N_{p-1}=N_{s 0} / N_{p 0_{1}}=0.025$, $t_{0}=\frac{\mu t}{N_{p 0_{2}}}$.

Теперь рассмотрим эволюцию системы в случае, когда в начальный момент времени $N_{p 0_{1}}>N_{p 0_{2}}$ и определенности ради $N_{s 0}<N_{i 0}$. Решение уравнения (5) получаем в виде

$$
\begin{aligned}
N_{p_{1}}= & \frac{\left(N_{p 0_{1}}-N_{p 0_{2}}\right)\left(N_{p 0_{1}}+N_{i 0}\right)}{N_{p 0_{1}}-N_{p 0_{2}}-\left(N_{p 0_{2}}+N_{i 0}\right)}, \\
& \times s n^{2}\left( \pm \sqrt{\left(N_{p 0_{2}}+N_{s 0}\right)\left(N_{p 0_{1}}+N_{i 0}\right)} \mu t+F\left(\varphi_{0}, k\right)\right)
\end{aligned}
$$

где величины $k$ и $\varphi_{0}$ выражаются формулами

$$
\begin{aligned}
k^{2} & =\frac{\left(N_{p 0_{1}}+N_{s 0}\right)\left(N_{p 0_{2}}+N_{i 0}\right)}{\left(N_{p 0_{2}}+N_{s 0}\right)\left(N_{p 0_{1}}+N_{i 0}\right)}, \\
\varphi_{0} & =\arcsin \sqrt{\frac{\left(N_{p 0_{1}}+N_{i 0}\right) N_{p 0_{2}}}{\left(N_{p 0_{2}}+N_{i 0}\right) N_{p 0_{1}}}} .
\end{aligned}
$$

Из (16) легко получить период $T$ колебаний плотности поляритонов накачки:

$$
T=2 K(k) /\left(\mu \sqrt{\left(N_{p 0_{2}}+N_{s 0}\right)\left(N_{p 0_{1}}+N_{i 0}\right)}\right) .
$$

В данном случае эволюция системы представляет собой периодическое превращение поляритонов накачки в поляритоны сигнальной и холостой мод, и обратно (рис. 5).

Наконец, если положить $N_{p 0_{1}}=N_{p 0_{2}}$, то решение уравнения (5) получается в виде

$$
N_{p_{1}}=\frac{4 a b}{C \exp ( \pm 2 \mu \sqrt{a b} t)+2(a+b)+\frac{(a-b)^{2}}{C} \exp (\mp 2 \mu \sqrt{a b} t)},
$$

где

$$
\begin{gathered}
a=N_{p 0_{1}}+N_{s 0}, \quad b=N_{p 0_{1}}+N_{i 0}, \\
C=\frac{\left(\sqrt{N_{i 0}\left(N_{p 0_{1}}+N_{s 0}\right)+N_{s 0}\left(N_{p 0_{1}}+N_{i 0}\right)}\right)}{N_{p 0_{1}}},
\end{gathered}
$$




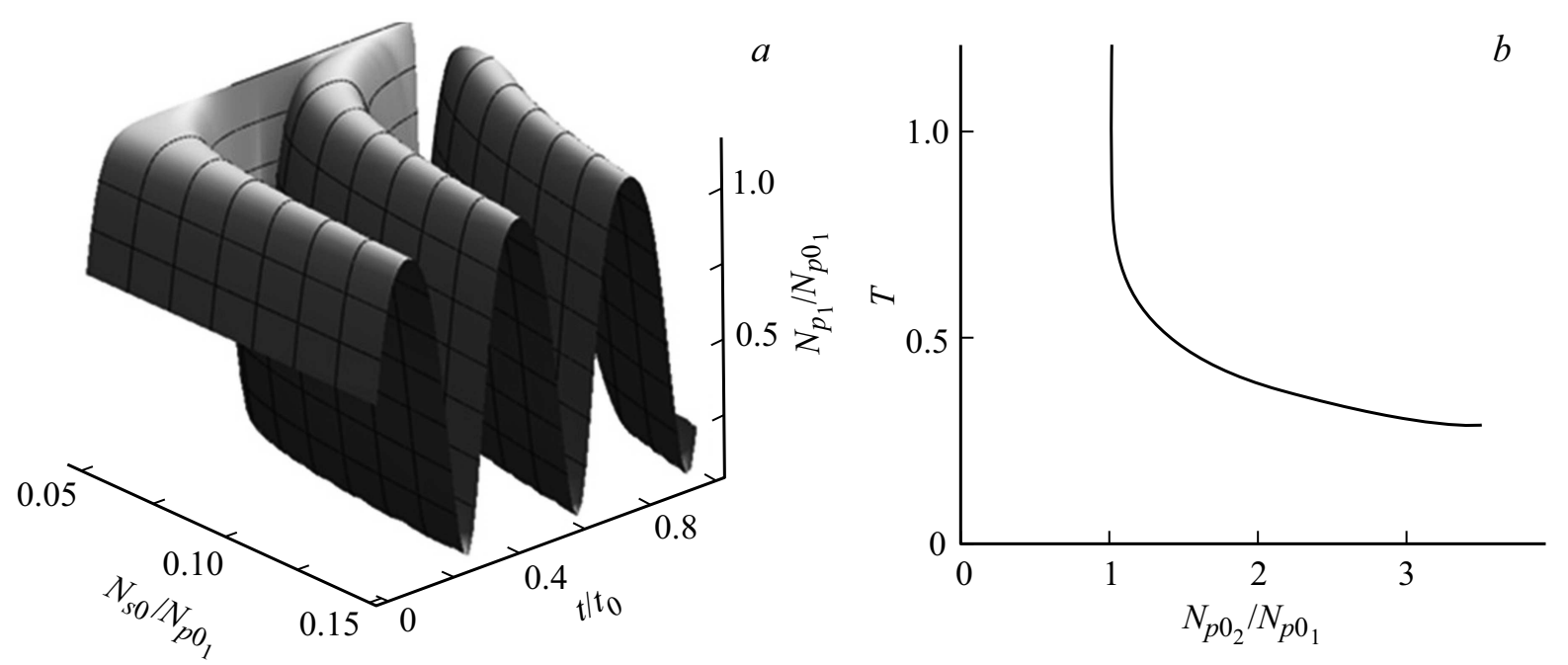

Рис. 5. (а) Временная эволюция нормированной плотности поляритонов накачки $N_{p_{1}} / N_{p 0_{1}}$ в зависимости от отношения начальных концентраций $N_{s 0} / N_{p 0_{1}}$ при $N_{p 0_{2}} / N_{p 0_{1}}=1.2$ и $N_{i 0} / N_{p 0_{1}}=0.05, t_{0}=\frac{\mu t}{N_{p 0_{2}}} ;(b)$ период колебаний $T$ в зависимости от отношения начальных концентраций $N_{p 0_{2}} / N_{p 0_{1}}$.

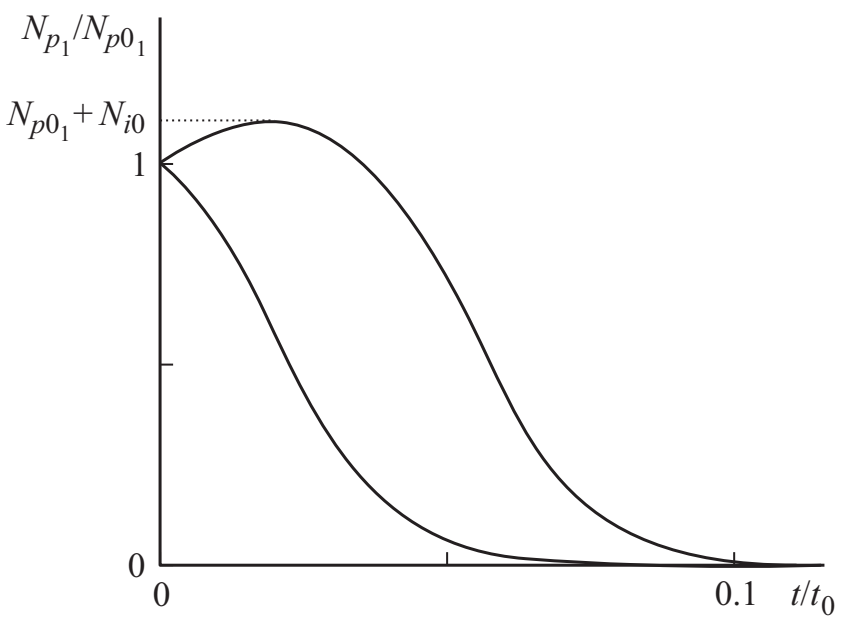

Рис. 6. Временная эволюция нормированной плотности поляритонов накачки $N_{p_{1}} / N_{p 0_{1}}$ при $N_{i 0} / N_{p 0_{1}}=0.1, N_{s 0} / N_{p 0_{1}}=0.25$, $t_{0}=\frac{\mu t}{N_{p 0_{2}}}$.

и при $N_{s 0}=N_{i 0}$ получаем

$$
N_{p_{1}}=\frac{N_{p 0_{1}}\left(N_{p 0_{1}}+N_{s 0}\right) \exp \left( \pm 2 \mu t\left(N_{p 0_{1}}+N_{s 0}\right)\right)}{N_{s 0}+N_{p 0_{1}} \exp \left( \pm 2 \mu\left(N_{p 0_{1}}+N_{s 0}\right)\right)} .
$$

На рис. 6 представлена временная эволюция поляритонов накачки в зависимости от нормированной начальной плотности поляритонов накачки. Видно, что в случае решения со знаком $(+)$ все поляритоны накачки $N_{p_{1}}$ превращаются в поляритоны сигнальной и холостой мод, чем эволюция и завершается. Что касается решения со знаком (-), то вначале плотность поляритонов накачки $N_{p_{1}}$ увеличивается, достигая своего максимального значения $N_{p 0_{1}}+N_{i 0}$, а затем монотонно уменьшается.
Видно, что на больших временах оба решения ведут себя одинаково. Таким образом, в данном случае получаем апериодический режим эволюции, сводящийся к тому, что все поляритоны накачки превращаются в поляритоны сигнальной и холостой мод. Скорость изменения плотности поляритонов определяется их начальной концентрацией.

\section{Заключение}

Таким образом, при накачке нижней поляритонной ветви в двух близких точках возможен преимущественно периодический процесс превращения пары поляритонов накачки в поляритоны сигнальной и холостой мод. Амплитуда и период колебаний плотности поляритонов определяются начальными условиями. Бо́льшая из начальных плотностей поляритонов накачки определяет период колебаний, а меньшая - амплитуду. Таким образом, введение двух независимых накачек приводит к увеличению степеней свободы системы. В свою очередь, это приводит к возможности введения двух различных приближений заданной плотности поляритонов накачки и к введению, вообще говоря, процессов трехволнового, а не четырехволнового взаимодействий, а также к более гибкому управлению динамикой параметрических осцилляций с помощью двух накачек $N_{p 0_{1}}$ и $N_{p 0_{2}}$. Необходимо отметить, что при накачке нижней поляритонной ветви в одной точке кривой дисперсии в условиях точного резонанса при начальной разности фаз, равной $\theta_{0}=\frac{\pi}{2}$, наблюдался только апериодический режим эволюции превращения пары поляритонов накачки в поляритоны сигнальной и холостой мод [34-38]. 


\section{Список литературы}

[1] Kavokin A.V., Malpuech G. Thin Films and Nanostructures. Cavity polaritons. 2003. 2043 p.

[2] Deng H., Haug H., Yamamoto Y. // Rev. Mod. Phys. 2010. V. 82. P. 1489.

[3] Kavokin A. // Appl. Phys. A. 2007. V. 89. P. 241.

[4] Glazov M.M., Kavokin K.V. // Phys. Rev. B. 2006. V. 73. P. 245317.

[5] Shelykh I.A., Johne R., Solnyshkov D.D., Kavokin A.V., Gippius N.A., Malpuech G. // Phys. Rev. B. 2007. V. 76. P. 155308.

[6] Whittaker D.M. // Phys. Rev. B. 2001. V. 63. P. 193305.

[7] Ciuti C., Schwendimann P., Deveaud B., Quattropani A. // Phys. Rev. B. 2000. V. 62. P. R4825.

[8] Savvidis P.G., Baumberg J.J., Stevenson R.M., Skolnick M.S., Whittaker D.M., Roberts J.S. // Phys. Rev. Lett. 2000. V. 84. P. 1547.

[9] Baumberg J.J., Savvidis P.G., Stevenson R.M., Tartakovskii A.I., Skolnick M.S., Whittaker D.M., Roberts J.S. // Phys. Rev. B. 2000. V. 62. P. R16247.

[10] Ciuti C. // Phys. Rev. B. 2004. V. 69. P. 245304.

[11] Schwendimann P., Ciuti C., Quattropani A. // Phys. Rev. B. 2003. V. 68. P. 165324.

[12] Savvidis P.G., Baumberg J.J., Porras D., Whittaker D.M., Skolnick M.S., Roberts J.S. // Phys. Rev. B. 2002. V. 65. P. 073309.

[13] Shelykh I.A., Kavokin A.V., Malpuech G. // Phys. St. Sol. B. 2005. V. 242. P. 2271.

[14] Stevenson R.M., Astratov V.N., Skolnick M.S., Whittaker D.M., Emam-Ismail M., Tartakovskii A.I., Savvidis P.G., Baumberg J.J., Roberts J.S. // Phys. Rev. Lett. 2000. V. 85. P. 3680.

[15] Tartakovskii A.I., Krzhizhanovskii D.N., Malpuech G., Emam-Ismail M., Chernenko A.V., Kavokin A.V., Kulakovskii V.D., Skolnick M.S., Roberts J.S. // Phys. Rev. B. 2003. V. 67. P. 165302.

[16] Tartakovskii A.I., Krzhizhanovskii D.N., Kulakovskii V.D. // Phys. Rev. B. 2000. V. 62. P. R13298.

[17] Ciuti C., Schwendimann P., Deveaud B., Quattropani A. // Phys. Rev. B. 2001. V. 63. P. 041303.

[18] Savvidis P.G., Ciuti C., Baumberg J.J., Whittaker D.M., Skolnik M.S., Roberts J.S. // Phys. Rev. B. 2001. V. 64. P. 075311.

[19] Savona V., Schwendimann P., Quattropani A. // Phys. Rev. B. 2005. V. 71. P. 125315.

[20] Kavokin A., Lagoudakis P.G., Malpuech G., Baumberg J.J. // Phys. Rev. B. 2003. V. 67. P. 195321.

[21] Saba M., Ciuti C., Bloch J., Tierry-Mieg V., Adre R., Dang L.S., Kundermann S., Mura A., Bongiovanni'C., Staehli J.E., Deveaud B. // Nature. 2001. V. 414. P. 731.

[22] Huang R., Tassone F., Yamamoto Y. // Phys. Rev. B. 2000. V. 61. P. R7854.

[23] Baas A., Karr J.-Ph., Romanelli M., Bramati A., Giacobino E. // Phys. Rev. B. 2004. V. 70. P. R161307.

[24] Baas A., Karr J.-Ph., Eleuch H., Giacobino E. // Phys. Rev. A. 2004. V. 69. P. 023819.

[25] Krizhanovski D.N., Gavrilov S.S., Love A.P.D., Sanvitto D., Gippius N.A., Tikhodeev S.G., Kulakovskii V.D., Whittaker D.M., Skolnick M.S., Roberts J.S. // Phys. Rev. B. 2008. V. 77. P. 115336.
[26] Lagoudakis P.G., Savvidis P.G., Baumberg J.J., Whittaker D.M., Eastham P.R., Skolnick M.S., Roberts J.S. // Phys. Rev. B. 2002. V. 65. P. R161310.

[27] Tartakovskii A.I., Krzhizhanovskii D.N., Kurysh D.A., Kulakovskii V.D., Skolnick M.S., Roberts J.S. // Phys. Rev. B. 2202. V. 65. P. R081308.

[28] Gippius N.A., Tikhodeev S.G. // J. Phys.: Cond. Matt. 2004. V. 16. P. S3653.

[29] Agranovich V.M., Litinskaia M., Lidzey D.G. // Phys. St. Sol. B. 2002. V. 234. P. 130.

[30] Ciuti C. // Phys. Rev. B. 2004. V. 69. P. 245304.

[31] Oka H., Ishihara H. // Phys. Rev. Lett. 2008. V. 100. P. 170505.

[32] Shelykh I.A., Solnyshkov D.D., Pavlovic G., Malpuech G. // Phys. Rev. B. 2008. V. 78. P. 041302.

[33] Lagoudakis K.G., Pietka B., Wouters M., Andre R., DeveaudPledran B. // Phys. Rev. Lett. 2010. V. 105. P. 120403.

[34] Хаджи П.И., Васильева О.Ф. // Опт. и спектр. 2011. Т. 111. C. 831.

[35] Хаджи П.И., Васильева О.Ф. // ФТТ. 2011. Т. 53. С. 1216.

[36] Васильева О.Ф., Хаджси П.И. // Опт. и спектр. 2013. Т. 115. C. 922.

[37] Khadzhi P.I., Vasilieva O.F. // J. Nanophoton. 2012. V. 6. P. 061805.

[38] Khadzhi P.I., Vasilieva O.F. // J. Nanoelectron. Optoelectron. 2014. V. 9. P. 1.

[39] Mc Konstrie C.J., Radic S., Raymer M.G. // Opt. Express. 2004. V. 12. P. 5037.

[40] Okawachi Y., Yu M., Luke K., Carvalho D.O., Ramelow S., Farsi A., Lipson M., Gaeta A.L. // Opt. Lett. 2015. V. 40. P. 5267.

[41] Питаевский Л.П. // УФН. 1998. Т. 168. С. 641.

[42] Градштейн И.С., Рыжик И.М. Таблицы интегралов, сумм, рядов и произведений. М.: ГИФМЛ, 1963. 1100 с.

[43] Корн Г., Корн Т. Справочник по математике для научных работников и инженеров. М.: Наука, 1971. 832 с. 